\title{
Nanostructures for Efficient Hole Transport in Poly(3- hexylthiophene) Film: A Study by Conductive Atomic Force Microscopy
}

\section{$\operatorname{AUTHOR}(\mathrm{S})$ :}

Osaka, Miki; Benten, Hiroaki; Ohkita, Hideo; Ito, Shinzaburo; Ogawa, Hiroki; Kanaya, Toshiji

\section{CITATION:}

Osaka, Miki ...[et al]. Nanostructures for Efficient Hole Transport in Poly(3-hexylthiophene) Film: A Study by Conductive Atomic Force Microscopy. Journal of Physical Chemistry C 2015, 119(43): 24307-24314

\section{ISSUE DATE:}

2015-10-12

URL:

http://hdl.handle.net/2433/217958

\section{RIGHT:}

This document is the Accepted Manuscript version of a Published Work that appeared in final form in 'Journal of Physical Chemistry C', copyright @ A American Chemical Society after peer review and technical editing by the publisher. To access the final edited and published work see http://doi.org/10.1021/acs.jpcc.5b08237.; この論文は出版社版であり ません。引用の際には出版社版をご確認ご利用ください。; This is not the published version. Please cite only the published version. 


\section{Nanostructures for Efficient Hole Transport in Poly(3-hexylthiophene) Film: A Study by Conductive Atomic Force Microscopy}

Miki Osaka, ${ }^{1}$ Hiroaki Benten, ${ }^{* 1}$ Hideo Ohkita, ${ }^{1}$ Shinzaburo Ito, ${ }^{1}$ Hiroki Ogawa, ${ }^{2}$ and Toshiji Kanaya $^{2}$

${ }^{1}$ Department of Polymer Chemistry, Graduate School of Engineering, Kyoto University, Katsura, Nishikyo, Kyoto 615-8510, Japan

${ }^{2}$ Institute for Chemical Research, Kyoto University, Uji 611-0011, Japan

osaka@photo.polym.kyoto-u.ac.jp, benten@photo.polym.kyoto-u.ac.jp

ohkita@photo.polym.kyoto-u.ac.jp, sito@photo.polym.kyoto-u.ac.jp

ogawa@scl.kyoto-u.ac.jp, kanaya@scl.kyoto-u.ac.jp

Corresponding Author Footnote.

*Hiroaki Benten

Tel.: +81 753832614 Fax: +81 753832617 Email: benten@photo.polym.kyoto-u.ac.jp 
ABSTRACT: The growth of hole-transporting nanostructures of regioregular poly(3-hexylthiophene) (P3HT) films with thermal annealing were examined by conductive atomic force microscopy (C-AFM). The C-AFM current images visualized spatially inhomogeneous hole transport in the film on a nanometer scale, with relatively low conductive regions and high conductive domains. The high conductive domains were attributed to the relatively high density regions of P3HT nanocrystallites in the film, which were determined during spin-coating. The current images obtained from the same area of the P3HT film showed that thermal annealing improved the hole-transporting property of the film on average, but both the size and spatial distributions of the relatively high conductive domains in the as-spun film remained almost the same even after annealing. Furthermore, we found that the increase in current flow proceeded mostly in the relatively high conductive domains. In these domains, the electrical connectivity among the crystalline phases was effectively improved by the growth of individual nanocrystallites, leading to the formation of preferred hole-transporting pathways in the direction of film thickness. 


\section{INTRODUCTION}

Regioregular poly(3-hexylthiophene) (P3HT) is one of the most widely used semiconducting polymers as a key material for electronic applications such as organic field-effect transistors (OFETs) and solar cells owing to the excellent hole-transporting property of the film in both lateral and vertical directions. ${ }^{1-3}$ The efficient hole transport of P3HT is due to the material's strong self-organizing tendency to form crystallites by $\pi-\pi$ interchain stacking. On the other hand, such semicrystalline structures of P3HT films lead to performance variations, as is observed when preparing films under different film-processing conditions (e.g., spin-coating solvents, spin speed) ${ }^{4-9}$ and post-deposition treatments such as thermal annealing, ${ }^{10-13}$ as well as the molecular weights and regioregularity of polymer chains.,10,14-17 For example, morphology-dependent hole transport parallel to the layer plane has been reported for many OFETs. $^{4-8,10-12}$ The vertical hole transport throughout the layer thickness, which is directly related to the device performance of polymer-based solar cells, is also influenced by the semicrystalline structures. ${ }^{16,17}$ In particular, the growth of crystalline nanodomains by thermal annealing is believed to play a critical role in the increase in vertical hole transport in a film.

The size and orientation distribution of the P3HT nanocrystallites and the compositions of ordered and disordered phases in the film have been studied using characterization techniques such as X-ray diffraction (XRD), transmission electron microscopy, and atomic force microscopy (AFM). However, the interplay between the nanostructures and the macroscopic hole transport in the film is still far from being clearly understood. This is partly because even though these characterization techniques provide detailed structural information on the film, they do not provide direct information on the electronic properties of the individual nanostructures and their mutual electrical connectivity.

Conductive AFM (C-AFM) allows one to observe the electrical characteristics related to 
microstructures of materials with nanometer-scale resolution. ${ }^{18-26}$ We have previously demonstrated that the C-AFM approach visualizes the conductive nanodomains in the $\mathrm{P} 3 \mathrm{HT}$ films, and we showed that the conductive domains are essential for obtaining sufficient macroscopic hole transport in the film. In this study, we track the formation of characteristic nanostructures resulting from thermal annealing for sufficient hole transport to investigate the role of the growth of $\mathrm{P} 3 \mathrm{HT}$ nanocrystallites in the vertical hole transport in the films. To this end, we employed a conductive atomic force microscope equipped with a heating unit at the sample stage, which enabled us to obtain current images at the same location of the film before and after thermal annealing. In addition, the crystalline structure of P3HT in the film was characterized using grazing-incidence wide-angle X-ray scattering (GIWAXS).

\section{EXPERIMENTAL}

2.1. Materials. Regioregular P3HT was purchased from Aldrich (Figure 1). The head-to-tail regioregularity (RR), weight-average molecular weight $\left(M_{\mathrm{w}}\right)$, and polydispersity index (PDI, given by $M_{\mathrm{w}} / M_{\mathrm{n}}$, where $M_{\mathrm{n}}$ is the number-average molecular weight) provided by the Certificate of Analysis were $90 \%, 43,200 \mathrm{~g} \mathrm{~mol}{ }^{-1}$, and 1.9, respectively. Poly(3,4-ethylenedioxythiophene):poly(4-styrenesulfonate) (PEDOT:PSS) was purchased from H.C. Stark (PH500).
(a) $\mathrm{P} 3 \mathrm{HT}$
(b) for absorption
(c) for $J-V$ and C-AFM
(d) for GIWAXS

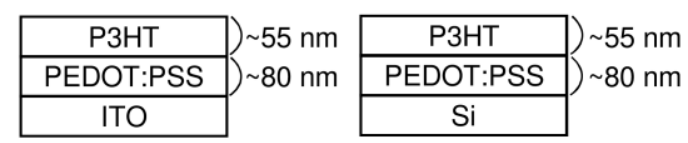

Figure 1. (a) Chemical structure of P3HT. Illustration of sample structures for (b) UV-visible absorption, (c) macroscopic $J-V$ and C-AFM, and (d) GIWAXS measurements. 
2.2. Sample Preparation. An indium-tin-oxide substrate (ITO) (flat ITO, GEOMATEC, Japan), an Si(100) wafer (Yamanaka Hutech, Japan), and a quartz substrate were sequentially washed for 15 min by ultrasonication with toluene, acetone, and ethanol, and then dried with an $\mathrm{N}_{2}$ flow. The cleaned substrates were further treated with a $\mathrm{UV}-\mathrm{O}_{3}$ cleaner (NL-UV2535, Nippon Laser \& Electronics Lab., Japan) for 30 min. An 80-nm-thick PEDOT:PSS layer was spin-coated at $400 \mathrm{rpm}$ for $10 \mathrm{~s}$ and $3000 \mathrm{rpm}$ for $99 \mathrm{~s}$ onto the ITO substrate and the Si wafer, and dried at $140{ }^{\circ} \mathrm{C}$ for $30 \mathrm{~min}$ in air. $\mathrm{P} 3 \mathrm{HT}$ films were prepared by spin-coating a 10 $\mathrm{mg} \mathrm{mL} \mathrm{m}^{-1}$ solution in chloroform at a spinning rate of $3000 \mathrm{rpm}$ for $60 \mathrm{~s}$ onto the PEDOT:PSS/ITO (for macroscopic $J-V$ and C-AFM measurements), PEDOT:PSS/Si (for GIWAXS measurements), and quartz (for absorption measurements) substrates (Figure 1). The thickness of the P3HT films was $\sim 55 \mathrm{~nm}$; it was determined by contact-mode AFM measurement (SPM-9600, Shimadzu, Japan) as follows: a part of the film was scratched with a sharp needle to expose the substrate, and the film thickness was evaluated from the difference between the heights of the film and substrate surface. We note that the vibronic structures identified in the absorption spectrum of the P3HT film spin-coated onto the PEDOT:PSS-coated substrate were almost identical to those in the spectrum of a film spin-coated onto quartz (see supporting information, Fig. S1).

2.3. Macroscopic $\boldsymbol{J}-\boldsymbol{V}$ Measurements. The hole-only current density-voltage $(J-V)$ characteristics were measured for the P3HT films sandwiched by the PEDOT:PSS/ITO substrate and an Au electrode. The P3HT layer was spin-coated onto the PEDOT:PSS/ITO substrate and then annealed at a different temperature $\left(100,140\right.$, or $\left.180{ }^{\circ} \mathrm{C}\right)$ for $10 \mathrm{~min}$ in an $\mathrm{N}_{2}$ atmosphere. Finally, the Au top electrode with a thickness of $70 \mathrm{~nm}$ and a surface area of $0.07 \mathrm{~cm}^{2}$ was vacuum-deposited at $3 \times 10^{-4} \mathrm{~Pa}$ on top of the P3HT film. The $J-V$ characteristics were measured with a direct-current voltage and a current source/monitor (R6243, Advantest) in the dark at room temperature. The conductivity $\sigma$ was calculated 
from the slope of the $J-V$ curve at lower voltages given by Ohm's law, $J_{\mathrm{OHM}}=\sigma V / L$, where $V$ is the applied voltage and $L$ is the sample thickness. The hole mobility $\mu_{\mathrm{h}}$ was determined by fitting the space-charge-limited current (SCLC) that appeared at higher voltages with the Mott-Gurney equation, $J_{\mathrm{SCL}}=(9 / 8) \varepsilon_{0} \varepsilon_{\mathrm{r}} \mu_{\mathrm{h}} V^{2} / L^{3}$, where $\varepsilon_{0}$ is the vacuum permittivity and $\varepsilon_{\mathrm{r}}$ is the dielectric constant of the film (we assumed $\varepsilon_{\mathrm{r}}=3$ for P3HT). ${ }^{27-29}$

2.4. C-AFM Measurements. Figure 2a shows an illustration of the set-up for the conductive atomic force microscope employed in this study. C-AFM measurements were performed using the microscope (SPM-9600, Shimadzu, Japan) in contact mode with an Au-coated silicon probe (PPP-CONTAu, NANOSENSORS ${ }^{\mathrm{TM}}$, Switzerland; tip radius $<50 \mathrm{~nm}$; spring constant $=0.2-0.25 \mathrm{~N} \mathrm{~m}^{-1}$ ). Sample heating was performed using the sample heating unit (Shimadzu, Japan): a heating holder was sandwiched between the sample and the piezoelectric element of the C-AFM, and the sample was heated by the heater incorporated inside the heating holder. A sample bias was applied to the ITO substrate and the probe was grounded. The surface topography and corresponding current images were simultaneously obtained by C-AFM measurements operating at a constant sample bias of $+1.0 \mathrm{~V}$. All C-AFM measurements were performed under an $\mathrm{N}_{2}$ atmosphere using a controlled-environment chamber (CH-III, Shimadzu, Japan). 
(a)

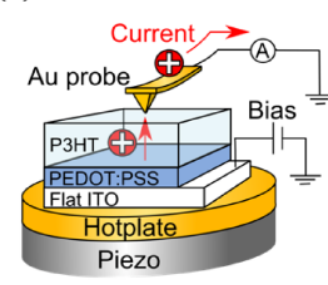

(b)

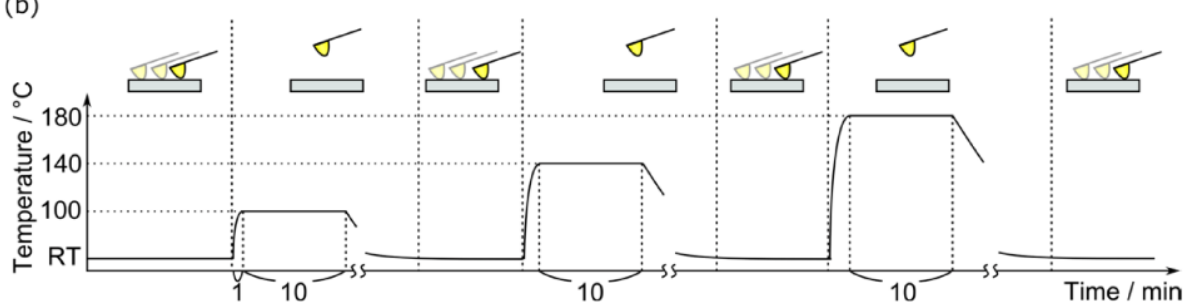

Figure 2. (a) Illustration of the conductive atomic force microscope employed in this study: a heating holder was sandwiched between the sample and the piezoelectronic element. A sample bias was applied to the ITO substrate and the probe was grounded. (b) Operating procedure of C-AFM fixed-point measurements. The measurements were operated at room temperature RT, and the sample was annealed by the underlying hot plate after the probe was released from the sample surface.

Figure $2 \mathrm{~b}$ shows the operating procedure of our C-AFM measurements. First, the unannealed (as-spun) film was measured. Then, after releasing the probe from the surface, the film was thermally annealed at $100{ }^{\circ} \mathrm{C}$ for $10 \mathrm{~min}$ by the heating holder under the sample. After natural cooling of the film to room temperature, the probe was placed on the film surface in the same region as that for the measurement before annealing, and the surface topology and a current image were obtained. After the measurement, the film was annealed at 140 and then $180{ }^{\circ} \mathrm{C}$ for $10 \mathrm{~min}$ at each temperature, and the images were obtained in the same manner after each round of annealing. These annealing and measurement cycles were carried out under an $\mathrm{N}_{2}$ atmosphere. The effect of stage drift during the annealing was corrected by using the coordinate data of grains in the topographical image as fiducial markers (see supporting information, Fig. S2), which enabled us to acquire C-AFM images at the same location of a film before and after annealing. The tip-sample contact force was kept to a minimum to prevent damages to the sample surface during the continuous scan (see supporting information, Fig. S3). We also noted that the average value of the C-AFM current showed very weak dependence on the number of the imaging cycles, which implied that the Au-coated probe did not wear out during the continuous imaging (see Supporting 
Information, Fig. S4).

2.5. GIWAXS Measurements. The GIWAXS measurements were carried out at room temperature for unannealed and annealed P3HT films using the BL03XU beam line of SPring-8 (Japan Synchrotron Radiation Research Institute, Hyogo, Japan). The wavelength $\lambda$ of the X-rays was set to $0.1 \mathrm{~nm}$ and the $\mathrm{X}$-ray beam was directed on the sample at an incident angle $\alpha_{\mathrm{i}}$ of $0.14^{\circ}$ with respect to the sample surface. The scattering signal was detected by a two-dimensional CCD camera with a $3000 \times 3000$ pixel imaging plate, which was located $464 \mathrm{~mm}$ from the sample. The scattering intensity was recorded as a function of the two scattering angles: in-plane angle $2 \theta_{\mathrm{f}}$ with respect to the plane of incidence and exit angle $\alpha_{\mathrm{f}}$ with respect to the sample surface. ${ }^{30}$ These angles are related to the scattering vector along the $q_{y^{-}}$and the $q_{z}$-axis according to the equations: $q_{y}=k_{0}\left[\sin \left(2 \theta_{\mathrm{f}}\right) \cos \left(\alpha_{\mathrm{f}}\right)\right], q_{z}=$ $k_{0}\left[\sin \left(\alpha_{\mathrm{f}}\right)+\sin \left(\alpha_{\mathrm{i}}\right)\right]$, and $k_{0}=2 \pi / \lambda^{30}$ The thermal annealing of the P3HT films for GIWAXS measurements was conducted at different temperatures $\left(100,140\right.$, or $\left.180{ }^{\circ} \mathrm{C}\right)$ for 10 min under an $\mathrm{N}_{2}$ atmosphere.

2.6. Absorption Measurements. The UV-visible absorption spectra of the 100-, 140-, and $180{ }^{\circ} \mathrm{C}$-annealed films were measured at room temperature after the films were thermally annealed under an $\mathrm{N}_{2}$ atmosphere at each temperature for $10 \mathrm{~min}$. The in situ temperature dependence of the absorption spectra was obtained for a film during heating at 100, 140, and $180{ }^{\circ} \mathrm{C}$ in a vacuum. The temperature of the P3HT film was controlled in a cryostat (OptistatDN-V, Oxford Instruments, UK) with a temperature control unit (ITC503S, Oxford Instruments, UK).

\section{RESULTS AND DISCUSSION}

3.1. Nanoscale Hole-Transporting Property of P3HT Films. We first characterized the 
changes in the macroscopic hole-transporting properties of the P3HT film that took place as the films were thermally annealed. The values of $\sigma$ and $\mu$ calculated for the unannealed and annealed P3HT films are listed in Table 1. Both $\sigma$ and $\mu$ values increased with increasing temperature, demonstrating that thermal annealing increased the hole-transporting capability of the P3HT film.

Table 1. Macroscopic conductivity ( $\sigma)$ and hole mobility $(\mu)$ of P3HT films

\begin{tabular}{ccccc}
\hline $\begin{array}{c}\text { Annealing } \\
\text { temperature }\left({ }^{\circ} \mathrm{C}\right)\end{array}$ & As-spun & 100 & 140 & 180 \\
$\sigma\left(10^{-7} \mathrm{~S} \mathrm{~cm}^{-1}\right)$ & 0.75 & 0.97 & 1.3 & 1.7 \\
$\mu\left(10^{-4} \mathrm{~cm}^{2} \mathrm{~V}^{-1} \mathrm{~s}^{-1}\right)$ & 1.1 & 1.6 & 2.0 & 2.7 \\
\hline
\end{tabular}

Figure 3 shows surface topographical images and the corresponding C-AFM current images for the same area of a P3HT film that was first unannealed and then annealed sequentially at 100 , then 140 , and finally $180{ }^{\circ} \mathrm{C}$ for $10 \mathrm{~min}$ at each temperature. No significant differences were observed in the topographical images before and after the annealing (Figure 3a-d). The cross-sectional height profiles along the broken lines in each image are shown in Figure 4; the surface structures were little changed by the annealing. Contrary to these topographical images, the corresponding current images (Figure $3 \mathrm{e}-\mathrm{h}$ ) show the growth in the hole-transporting capability of the P3HT film. Furthermore, the C-AFM current images reveal that the current magnitudes were not uniform but spatially inhomogeneous on the length scale of several tens to hundreds of nanometers. The mean values of the current $\left(I_{\mathrm{av}}\right)$ increased from 42 to $80 \mathrm{pA}$ and the values of the relative standard deviation of $I_{\mathrm{av}}$ (RSD) extracted from the current histograms increased from 11 to $22 \%$ with increasing temperature. It should be noted here that there was little direct correlation between the surface topographical structures and the current magnitudes (see Supporting Information, Fig. S5), suggesting that the spatial distributions of the local current were not 
associated with the topographical features of the film. The qualitatively similar changes in hole-transport distribution have been reported for the blended film of P3HT with the fullerene derivative phenyl- $\mathrm{C}_{61}$-butyric acid methyl ester $(\mathrm{PCBM}),{ }^{31}$ while we have now achieved at the same location before and after the annealing, which allows accurate quantification of the electrical properties upon thermal treatment. 
(a) as-spun

(b) $100{ }^{\circ} \mathrm{C}$

(c) $140{ }^{\circ} \mathrm{C}$

(d) $180^{\circ} \mathrm{C}$

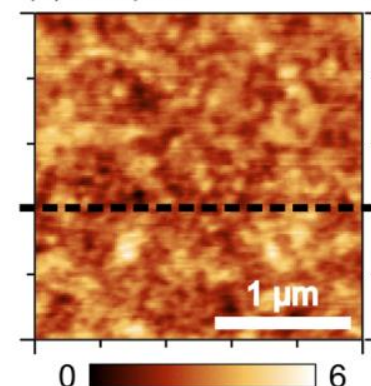

Height / nm
कास

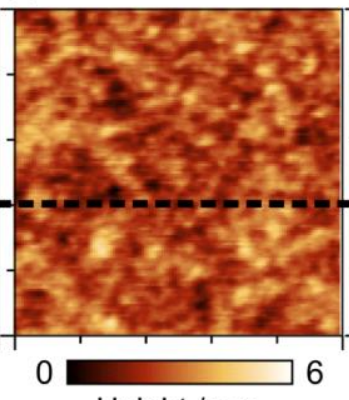

Height / nm

(f) $100^{\circ} \mathrm{C}$
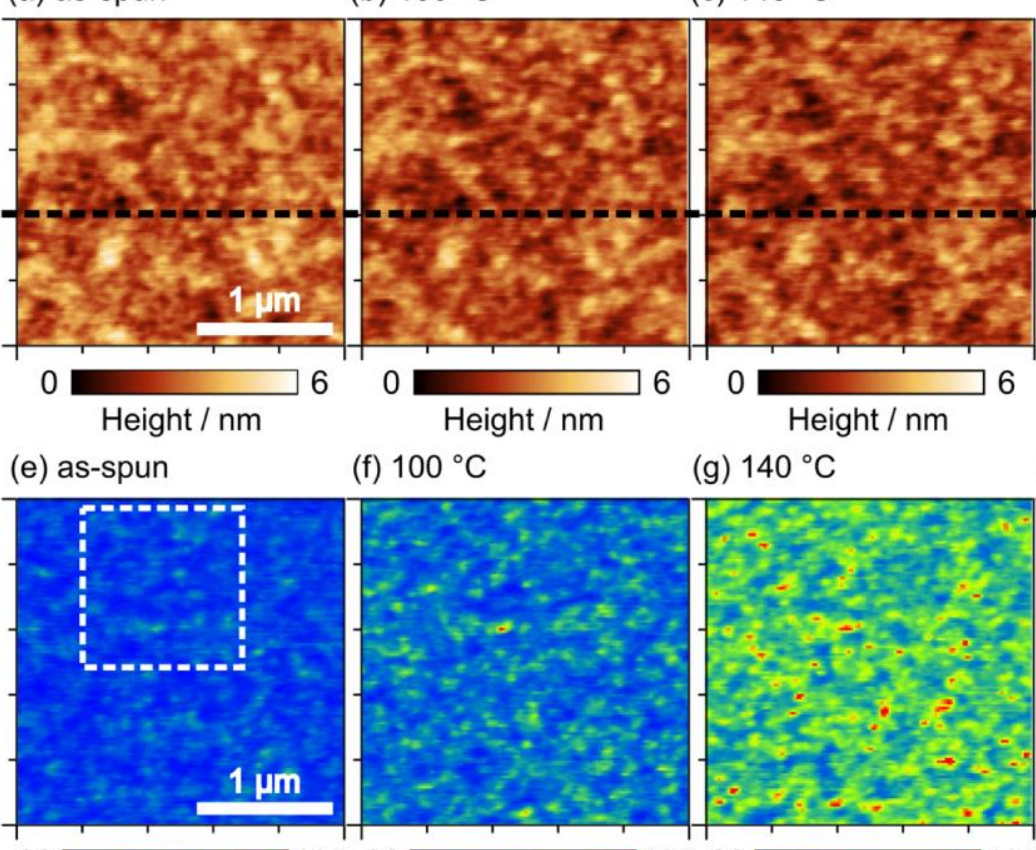

Height / nm

(g) $140^{\circ} \mathrm{C}$

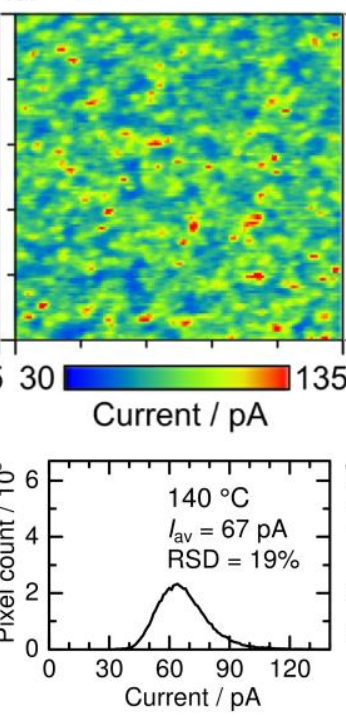

Current / pA

Current / pA

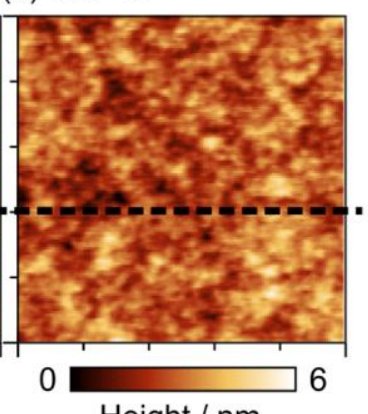

Height / nm

(h) $180^{\circ} \mathrm{C}$
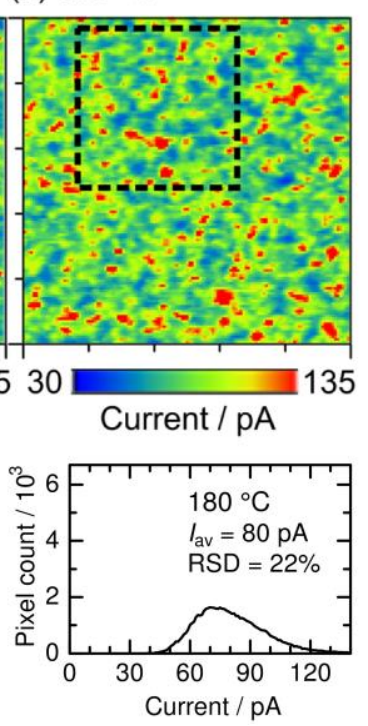

Figure 3. (a-d) Topographical and $(\mathrm{e}-\mathrm{h})$ current images $\left(2.5 \times 2.5 \mu \mathrm{m}^{2}\right)$ of a P3HT film that was (a, e) unannealed and then annealed sequentially at (b, f) $100{ }^{\circ} \mathrm{C}$, then (c, g) $140{ }^{\circ} \mathrm{C}$, and finally $(\mathrm{d}, \mathrm{h}) 180{ }^{\circ} \mathrm{C}$ for $10 \mathrm{~min}$ at each temperature. Histograms of the current images of P3HT film in $(\mathrm{e}-\mathrm{h})$ are shown beneath the images. $I_{\mathrm{av}}$ and RSD represent the average current and the relative standard deviation, respectively, extracted from the histograms.

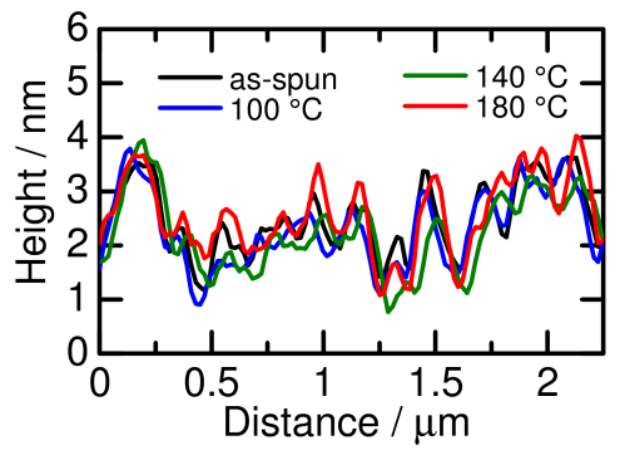

Figure 4. Cross-sectional profiles along the dashed lines in the topographical images in Figure $3 \mathrm{a}-\mathrm{d}$. 
We discuss in more detail the highly conductive nanostructures that appeared in the current images as a result of thermal annealing. Figure $5 \mathrm{a}$ and $\mathrm{b}$ show the magnified current images at the same locations in a film before and after annealing at $180{ }^{\circ} \mathrm{C}$ : they are marked by squares in Figure $3 \mathrm{e}$ and $\mathrm{h}$, respectively. Here, the color range used for the magnified images is individually rescaled: for both images, the relatively high current regions are represented as yellow and red, and the relatively low current regions as blue. As a result, the inhomogeneous hole-transporting capability of the film is clearly depicted both for the unannealed and annealed film. To compare the hole-transporting nanostructures, we outlined the relatively high and low current regions in black and white, respectively; these lines were drawn at the same position for both of these current images. This analysis reveals that spatial distributions of the relatively high and low current regions in the annealed film are well correlated with those in the unannealed film. The cross-sectional current profiles along the dashed lines in these images are shown in Figure 6a. The positions of the peaks and valleys are well matched between these two profiles. In addition, the lateral size of these relatively high conductive regions was determined to be approximately $200 \mathrm{~nm}$ regardless of annealing. We therefore conclude that the nanoscale morphologies responsible for the hole transport were structured during spin-coating of the film and were mostly preserved even after thermal annealing.

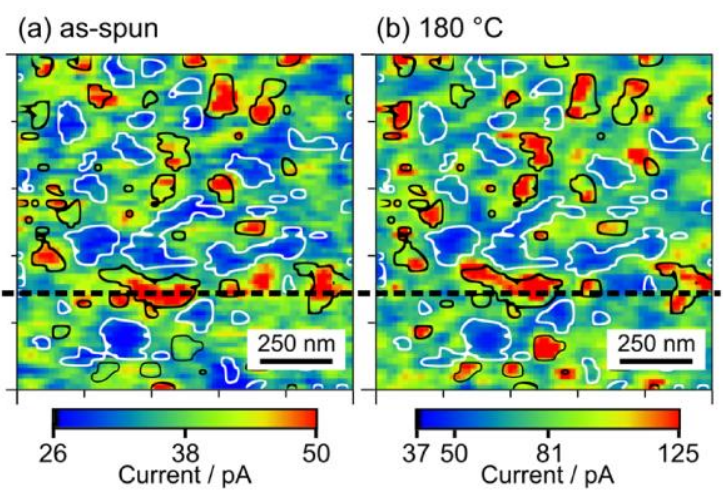

Figure 5. Current images of (a) the unannealed film and (b) the film annealed at $180{ }^{\circ} \mathrm{C}$, corresponding to the square areas marked in Figures $3 \mathrm{e}$ and $3 \mathrm{~h}$, respectively. 
(a)

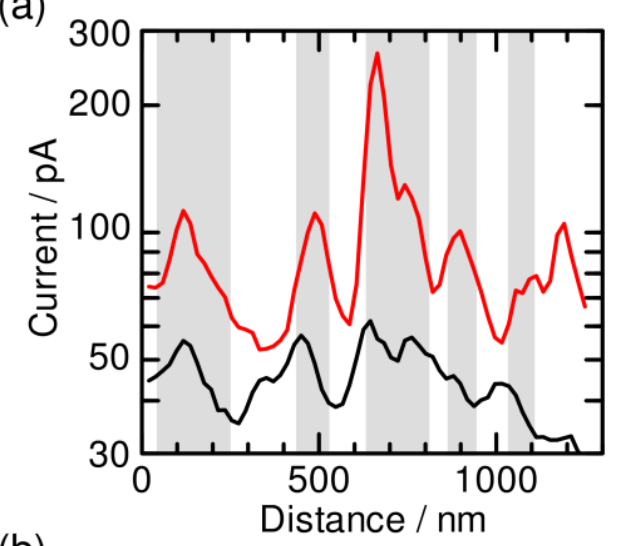

(b)

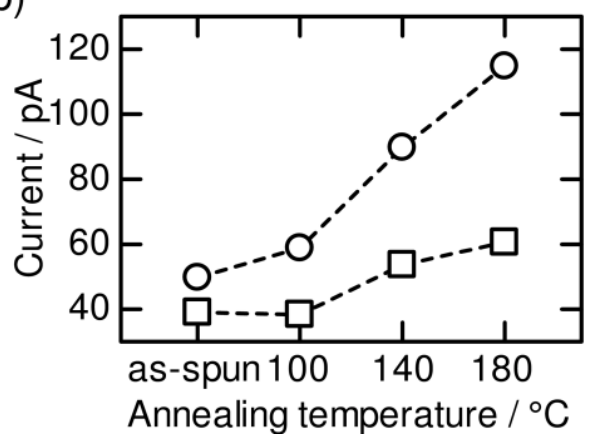

Figure 6. (a) Cross-sectional current profiles corresponding to the dashed lines in the images of Figure 5: the unannealed (black line) film and the film annealed at $180{ }^{\circ} \mathrm{C}$ (red line). (b) Annealing-temperature dependence of the amount of current that was carried through a peak within good conductive regions (circles) and poor conductive regions (squares).

We further examined the annealing-temperature dependence of the current magnitudes carried through the relatively high and low conductive regions in a P3HT film. Here, the current values at the peaks in the cross-sectional current profiles were all averaged and used as a representative of the relatively high conductive domains. On the other hand, the values corresponding to the valleys in the profiles were all averaged and used as a representative of the relatively low conductive regions. This analysis was conducted for the current images of $2.5 \times 2.5 \mu \mathrm{m}^{2}$ in Figure $3 \mathrm{e}-\mathrm{h}$ and the results are shown in Figure $6 \mathrm{~b}$; the current at the relatively high conductive domains increased by a factor of 2.3 with annealing at $180{ }^{\circ} \mathrm{C}$, whereas the increase in current from the relatively low conductive regions was limited by a factor of 1.5. These results demonstrate that the hole-transporting capability increased more 
effectively at the relatively high conductive domains than the surrounding low conductive regions. As a result, thermal annealing accentuated the differences in the hole-transporting capability in the film on the nanometer scale.

\subsection{Characterization of P3HT Crystalline Structures}

3.2.1. GIWAXS. To examine the correlation between the C-AFM current images and the P3HT crystalline structures in the film, GIWAXS measurements were performed. Figure 7a shows the 2D GIWAXS pattern of the unannealed film. Here, $q_{\mathrm{y}}$ is the component of the scattering vector parallel to the substrate plane, and $q_{\mathrm{z}}$ is the component perpendicular to the substrate plane. According to previous reports, the diffraction peak that appears at $q_{\mathrm{y}}=0.39$ $\AA^{-1}$ is assigned to the (100) reflection from the alkyl-stacking direction and the peak at $q_{\mathrm{z}}=$ $1.67 \AA^{-1}$ is assigned to the (010) reflection from the $\pi$-stacking direction as shown in Figure $7 b^{5,6,8,32}$

(a)

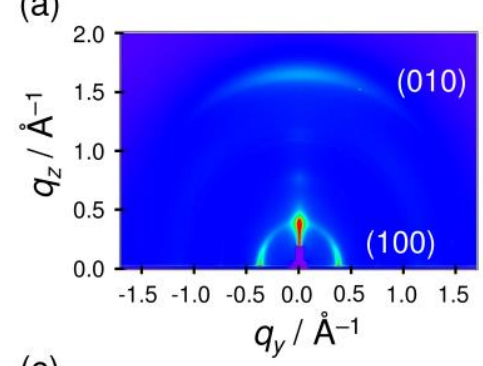

(c)

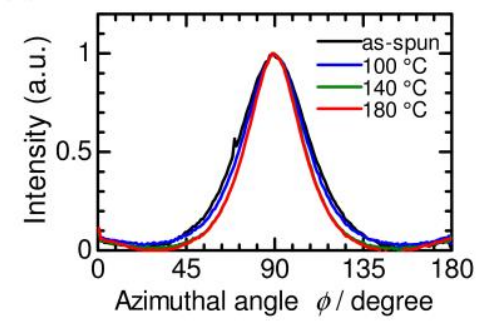

(b)

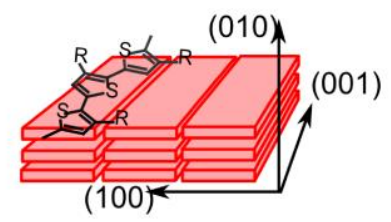

(d)

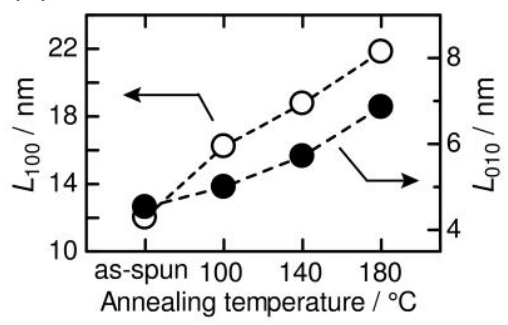

Figure 7. (a) Two-dimensional diffraction patterns of unannealed P3HT films. (b) Schematic representation of the orientations of the crystalline lamellae of P3HT. (c) Azimuthal profiles of the (010) $\pi$-stacking peak for the unannealed and annealed P3HT films. (d) Crystallite dimensions in the (010) $\pi$-stacking (solid circles) and (100) alkyl-stacking directions (open circles) as functions of the annealing temperature. 
The orientation of the crystals was determined from azimuthal dependence of the intensity of the (010) $\pi$-stacking peak. The (010) peak intensity obtained from the 2D GIWAXS image was plotted as a function of the azimuthal angle $(\phi)$ for the unannealed film and the films annealed at 100,140 , or $180{ }^{\circ} \mathrm{C}$ for $10 \mathrm{~min}$. As shown in Figure $7 \mathrm{c}$, all the films showed the (010) reflection peak at an angle of $\phi=90^{\circ}$, which corresponds to the scattering vector perpendicular to the substrate plane, indicating that the P3HT crystallites had predominantly face-on orientations in the films.

As to the face-on components of the crystallites, the dimensions in the (010) and (100) directions were calculated from the full width at half maximum (FWHM) of those Bragg peaks using Scherrer's equation (the shape factor $K$ was assumed to be 0.9$)^{33}$ and the results are plotted in Figure $7 \mathrm{~d}$ as functions of the annealing temperature. As a result of thermal annealing, the P3HT crystallites grew in size from 5 to $7 \mathrm{~nm}$ in the $\pi$-stacking direction (solid circles) and from 12 to $23 \mathrm{~nm}$ in the alkyl-stacking direction (open circles).

3.2.2. Temperature Dependence of Absorption Spectra. Figure $8 \mathrm{a}-\mathrm{d}$ show the UV-visible absorption spectra of the unannealed P3HT film and the films after annealing at 100, 140, and $180{ }^{\circ} \mathrm{C}$ for $10 \mathrm{~min}$. As clearly indicated by their spectral shapes in the wavelength range of 500 to $650 \mathrm{~nm}$, the shoulders at 550 and $600 \mathrm{~nm}$, which represent ordered structures of the P3HT chains, ${ }^{34-36}$ became more pronounced after annealing at higher temperatures. The growth of the vibronic structures shows that the crystallinity of the films was increased by thermal annealing. We next measured the UV-visible absorption spectra of the P3HT film during heating at temperatures from 100 to 180 and then $240{ }^{\circ} \mathrm{C}$ (Figure 9a). Here, the spectrum at $240{ }^{\circ} \mathrm{C}$ represents the absorption characteristics of amorphous P3HT because the temperature is higher than the melting temperature of P3HT crystals $\left(T_{\mathrm{m}}=220{ }^{\circ} \mathrm{C}\right) .{ }^{12,14,33}$ At 
room temperature, the film showed an absorption maximum at $532 \mathrm{~nm}$ and shoulders at 550 and $600 \mathrm{~nm}$. Upon heating, the absorption maximum was blue-shifted and the absorbance of these bands decreased continuously. This observation suggests a continuous disruption of ordered structures of the polymer chains. ${ }^{33,37}$ On the other hand, the absorption was still obvious at wavelengths between 550 and $650 \mathrm{~nm}$ when compared with the spectrum of amorphous P3HT (dashed line in Figure 9a), suggesting that P3HT crystallites remained in the film during the heating at temperatures below $180{ }^{\circ} \mathrm{C}$. To confirm this, the $\pi$-conjugation length $\left(L_{\pi}\right)$ of P3HT chains was estimated from the excitonic band widths $W$, obtained by analyzing the UV-visible absorption spectra in Figures $8 \mathrm{a}-\mathrm{d}$ and $9 \mathrm{a}$ on the basis of the weakly interacting H-aggregate model (see Supporting Information, Fig. S6). ${ }^{37,38}$ Here, $W$ is related to the length of the cofacially arranged chain segments in the crystallites. ${ }^{37,38}$ Using the simulation data from Gierschner et al. (see Supporting Information, Figure S7), we calculated the number of interacting repeating units (the number of planarized thiophene repeating units) $N$, from the excitonic bandwidths $W$ (see Supporting Information, Fig. S7). ${ }^{37,38}$ The value of $L_{\pi}$ was calculated by multiplying $N$ by the length of a P3HT repeating unit, $0.4 \mathrm{~nm}$. As shown in Figure $9 \mathrm{~b}, L_{\pi}$ decreased with increasing temperature but it still showed the value of $6.0 \mathrm{~nm}$ during heating at $180{ }^{\circ} \mathrm{C}$. Moreover, upon cooling to room temperature, $L_{\pi}$ became larger than the initial value of $7.0 \mathrm{~nm}$ before heating. These results imply that the ordering or crystallization of P3HT chains proceeded as illustrated in Figure 9c. During thermal annealing, part of the ordered structure of $\mathrm{P} 3 \mathrm{HT}$ chains was disrupted by activated relaxations of the P3HT chains, resulting in an increase in the disordered nature of the film. During cooling, the flexible P3HT chains around the remaining crystallites reorganized preferentially to form an extended ordered structure, enlarging the size of the crystallites, as shown by the GIWAXS analysis. 


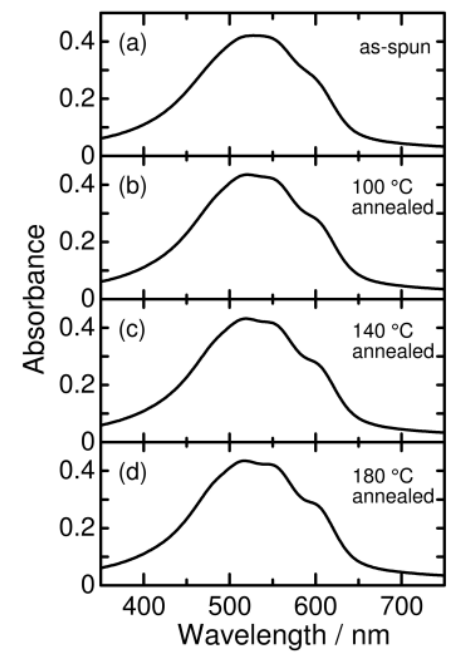

Figure 8. UV-visible absorption spectra of (a) the unannealed P3HT film and the films after annealing at (b) $100{ }^{\circ} \mathrm{C}$, (c) $140{ }^{\circ} \mathrm{C}$, and (d) $180{ }^{\circ} \mathrm{C}$ for $10 \mathrm{~min}$.

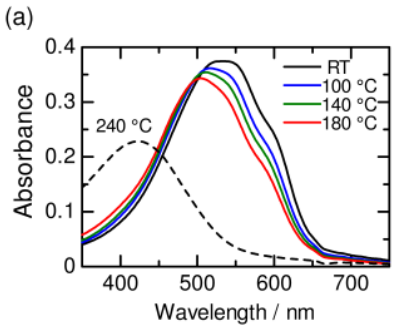

(b)
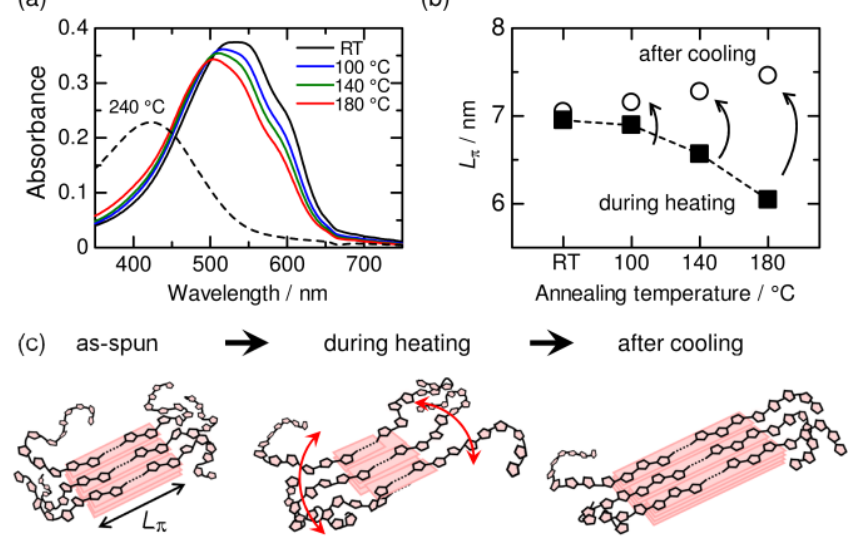

$\rightarrow \quad$ after cooling

Figure 9. (a) UV-visible absorption spectra of P3HT films measured at room temperature RT (black line), $100{ }^{\circ} \mathrm{C}$ (blue line), $140{ }^{\circ} \mathrm{C}$ (green line), $180{ }^{\circ} \mathrm{C}$ (red line), and $240{ }^{\circ} \mathrm{C}$ (dashed line). (b) $\pi$-conjugation length of P3HT chains during heating (squares) and after cooling (circles). (c) Illustration of ordering or crystallization of P3HT chains by thermal annealing.

\subsection{Correlation between Crystalline Structures and Hole Transport in the Film. Our} analysis of GIWAXS and absorption spectra showed that the P3HT crystallites grew in size as a result of thermal annealing, but the C-AFM current images revealed that there existed a large discrepancy in size between the individual P3HT crystallites and the relatively high conductive domains $(\sim 200 \mathrm{~nm})$. These results suggest that the aggregation of P3HT 
nanocrystallites, through the electrically connected networks among the crystallites, formed the high conductive domains. In other words, the relatively high and low conductive regions visualized in Figure 5 should be assigned to the relatively high and low density regions, respectively, of P3HT crystallites that were inhomogeneously dispersed in the film. Furthermore, the growth of nanocrystallites by thermal annealing is considered to have occurred while keeping their original locations in the film, whose structure was established during spin-coating. The scenario is brought to light by the C-AFM images showing that locations of the relatively high conductive domains in the annealed film were spatially correlated with the original locations before annealing. As illustrated in Figure 10, for the relatively high-density regions of crystallites in the film, the electrical connectivity between the crystallites should have been effectively improved even if the individual crystalline size grew by just several nanometers. On the other hand, at the low-density regions of crystallites, the growth of the crystalline size did not make an equal contribution to the increase in current because the crystallites remained largely disconnected from each other, resulting in charge-transporting bottlenecks due to the surrounding disordered phase. As a result, the current carried through the relatively high conductive regions in the unannealed film was increased largely by thermal annealing rather than the current through the low conductive regions.

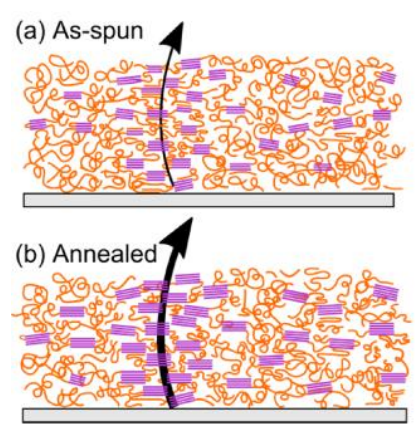

Figure 10. Illustration of the development of P3HT crystalline networks for efficient hole transport in a film. The P3HT crystallites are indicated by the purple rectangles. 


\section{CONCLUSION}

The growth of nanostructures for efficient hole transport in P3HT films was visualized by C-AFM. The C-AFM current images revealed that the capability of vertical hole transport of the film was not uniform but inhomogeneous on the nanometer scale, and the film consisted of relatively low conductive regions and high conductive domains with a typical size of approximately $200 \mathrm{~nm}$. These characteristic nanoscale morphologies responsible for the hole transport were structured during spin-coating of the film. Thermal annealing improved the hole-transporting property of the film on average, but both the size and spatial distributions of the relatively high conductive domains remained almost the same even after annealing. Furthermore, we found that the increase in the current flow proceeded mostly in the relatively high conductive domains rather than the surrounding low conductive regions, accentuating the nanoscale inhomogeneity of the hole-transporting capability in the film. The high conductive domains are attributed to the relatively high-density regions of $\mathrm{P} 3 \mathrm{HT}$ nanocrystallites dispersed in the film. For the domains, the electrical connectivity among the crystallites was effectively improved by the growth of individual crystallite size, leading to the formation of preferred hole-transporting pathways in the direction of film thickness. 


\section{Acknowledgments}

This work was supported by the CREST program of the Japan Science and Technology Agency, JSPS KAKENHI Grant Number 26288060, and Kansai Research Foundation for technology promotion. SR GISAWS measurements were carried out at the first hutch of the Consortium of Advanced Soft material Beamline (FSBL) with the proposal No. 2012B1971 and 2013B1881.

\section{Supporting Information Available}

Absorption spectra of P3HT film spin-coated on a quartz and a PEDOT:PSS-coated substrate, correction of thermal drift, damage of the film surface, dependence of the average C-AFM current on the number of imaging cycles, C-AFM cross-sectional current and height profiles, and estimation of $\pi$-conjugation length. This information is available free of charge via the Internet at http://pubs.acs.org. 


\section{References}

(1) Salleo, A. Charge Transport in Polymeric Transistors. Mater. Today 2007, 10, 38-45.

(2) Lim, J. A.; Liu, F.; Ferdous, S.; Muthukumar, M.; Briseno, A. L. Polymer Semiconductor Crystals. Mater. Today 2010, 13, 14-24.

(3) Salleo, A.; Kline, R. J.; DeLongchamp, D. M.; Chabinyc, M. L. Microstructural Characterization and Charge Transport in Thin Films of Conjugated Polymers. Adv. Mater. 2010, 22, 3812-3838.

(4) Chang, J.-F.; Sun, B.; Breiby, D. W.; Nielsen, M. M.; Sölling, T. I.; Giles, M.; McCulloch, I.; Sirringhaus, H. Enhanced Mobility of Poly(3-Hexylthiophene) Transistors by Spin-Coating from High-Boiling-Point Solvents. Chem. Mater. 2004, 16, 4772-4776.

(5) Kline, R. J.; McGehee, M. D.; Kadnikova, E. N.; Liu, J.; Fréchet, J. M. J.; Toney, M. F. Dependence of Regioregular Poly(3-Hexylthiophene) Film Morphology and Field-Effect Mobility on Molecular Weight. Macromolecules 2005, 38, 3312-3319.

(6) Kline, R. J.; McGehee, M. D.; Toney, M. F. Highly Oriented Crystals at the Buried Interface in Polythiophene Thin-Film Transistors. Nat. Mater. 2006, 5, 222-228.

(7) Joshi, S.; Grigorian, S.; Pietsch, U.; Pingel, P.; Zen, A.; Neher, D.; Scherf, U. Thickness Dependence of the Crystalline Structure and Hole Mobility in Thin Films of Low Molecular Weight Poly(3-Hexylthiophene). Macromolecules 2008, 41, 6800-6808.

(8) Duong, D. T.; Toney, M. F.; Salleo, A. Role of Confinement and Aggregation in Charge Transport in Semicrystalline Polythiophene Thin Films. Phys. Rev. B 2012, 86, 
205205.

(9) Vakhshouri, K.; Gomez, E. D. Effect of Crystallization Kinetics on Microstructure and Charge Transport of Polythiophenes. Macromol. Rapid Commun. 2012, 33, 2133-2137.

(10) Zen, A.; Pflaum, J.; Hirschmann, S.; Zhuang, W.; Jaiser, F.; Asawapirom, U.; Rabe, J. P.; Scherf, U.; Neher, D. Effect of Molecular Weight and Annealing of Poly(3-Hexylthiophene)s on the Performance of Organic Field-Effect Transistors. Adv. Funct. Mater. 2004, 14, 757-764.

(11) Cho, S.; Lee, K.; Yuen, J.; Wang, G.; Moses, D.; Heeger, A. J.; Surin, M.; Lazzaroni, R. Thermal Annealing-Induced Enhancement of the Field-Effect Mobility of Regioregular Poly(3-Hexylthiophene) Films. J. Appl. Phys. 2006, 100, 114503. Joshi, S.; Pingel, P.; Grigorian, S.; Panzner, T.; Pietsch, U.; Neher, D.; Forster, M.; Schere, U. Bimodal Temperature Behavior of Structure and Mobility in High Molecular Weight P3HT Thin Films. Macromolecules 2009, 42, 4651-4660.

(13) Salammal, S. T.; Mikayelyan, E.; Grigorian, S.; Pietsch, U.; Koenen, N.; Scherf, U.; Kayunkid, N.; Brinkmann, M. Impact of Thermal Annealing on the Semicrystalline Nanomorphology of Spin-Coated Thin Films of Regioregular Poly(3-Alkylthiophene)s as Observed by High-Resolution Transmission Electron Microscopy and Grazing Incidence X-Ray Diffraction. Macromolecules 2012, 45, 5575-5585.

(14) Zen, A.; Saphiannikova, M.; Neher, D.; Grenzer, J.; Grigorian, S.; Pietsch, U.; Asawapirom, U.; Janietz, S.; Scherf, U.; Lieberwirth, I.; et al. Effect of Molecular Weight on the Structure and Crystallinity of Poly(3-Hexylthiophene). Macromolecules 2006, 39, $2162-2171$. 
(15) Joshi, S.; Grigorian, S.; Pietsch, U. X-Ray Structural and Crystallinity Studies of Low and High Molecular Weight Poly(3-Hexylthiophene). Phys. Status Solidi 2008, 205, $488-496$.

(16) Kim, Y.; Cook, S.; Tuladhar, S. M.; Choulis, S. A.; Nelson, J.; Durrant, J. R.; Bradley,

D. D. C.; Giles, M.; McCulloch, I.; Ha, C.-S.; et al. A Strong Regioregularity Effect in Self-Organizing Conjugated Polymer Films and High-Efficiency Polythiophene:fullerene Solar Cells. Nat. Mater. 2006, 5, 197-203.

(17) Mauer, R.; Kastler, M.; Laquai, F. The Impact of Polymer Regioregularity on Charge Transport and Efficiency of P3HT:PCBM Photovoltaic Devices. Adv. Funct. Mater. 2010, 20, $2085-2092$.

De Wolf, P.; Snauwaert, J.; Clarysse, T.; Vandervorst, W.; Hellemans, L. Characterization of a Point-Contact on Silicon Using Force Microscopy-Supported Resistance Measurements. Appl. Phys. Lett. 1995, 66, 1530-1532.

(19) Shafai, C.; Thomson, D. J.; Simard-Normandin, M.; Mattiussi, G.; Scanlon, P. J. Delineation of Semiconductor Doping by Scanning Resistance Microscopy. Appl. Phys. Lett. 1994, 64, 342-344. Lin, H.-N.; Lin, H.-L.; Wang, S.-S.; Yu, L.-S.; Perng, G.-Y.; Chen, S.-A.; Chen, S.-H. Nanoscale Charge Transport in an Electroluminescent Polymer Investigated by Conducting Atomic Force Microscopy. Appl. Phys. Lett. 2002, 81, 2572-2574.

Alexeev, A.; Loos, J.; Koetse, M. M. Nanoscale Electrical Characterization of Semiconducting Polymer Blends by Conductive Atomic Force Microscopy (C-AFM). Ultramicroscopy 2006, 106, 191-199. 
(22) Groves, C.; Reid, O. G.; Ginger, D. S. Heterogeneity in Polymer Solar Cells: Local Morphology and Performance in Organic Photovoltaics Studied with Scanning Probe Microscopy. Acc. Chem. Res. 2010, 43, 612-620.

(23) Lee, L.-T.; Ito, S.; Benten, H.; Ohkita, H.; Mori, D. Current Mode Atomic Force Microscopy (C-AFM) Study for Local Electrical Characterization of Conjugated Polymer Blends. Ambio 2012, 41, 135-137.

(24) Osaka, M.; Benten, H.; Lee, L.-T.; Ohkita, H.; Ito, S. Development of Highly Conductive Nanodomains in Poly(3-Hexylthiophene) Films Studied by Conductive Atomic Force Microscopy. Polymer 2013, 54, 3443-3447.

(25) Wood, D.; Hancox, I.; Jones, T. S.; Wilson, N. R. Quantitative Nanoscale Mapping with Temperature Dependence of the Mechanical and Electrical Properties of Poly(3-Hexylthiophene) by Conductive Atomic Force Microscopy. J. Phys. Chem. C 2015, $119,11459-11467$.

(26) Kondo, Y.; Osaka, M.; Benten, H.; Ohkita, H.; Ito, S. Electron Transport Nanostructures of Conjugated Polymer Films Visualized by Conductive Atomic Force Microscopy. ACS Macro Lett. 2015, 4, 879-885.

(27) Blom, P. W. M.; de Jong, M. J. M.; Vleggaar, J. J. M. Electron and Hole Transport in Poly(p-Phenylene Vinylene) Devices. Appl. Phys. Lett. 1996, 68, 3308-3310.

(28) Malliaras, G. G.; Salem, J. R.; Brock, P. J.; Scott, C. Electrical Characteristics and Efficiency of Single-Layer Organic Light-Emitting Diodes. Phys. Rev. B 1998, 58, R13411-R13414. 
(29) Shen, Y.; Hosseini, A. R.; Wong, M. H.; Malliaras, G. G. How to Make Ohmic Contacts to Organic Semiconductors. ChemPhysChem 2004, 5, 16-25.

(30) Rauscher, M.; Paniago, R.; Metzger, H.; Kovats, Z.; Domke, J.; Peisl, J.; Pfannes, H.-D.; Schulze, J.; Eisele, I. Grazing Incidence Small Angle X-Ray Scattering from Free-Standing Nanostructures. J. Appl. Phys. 1999, 86, 6763-6769.

Pingree, L. S. C.; Reid, O. G.; Ginger, D. S. Imaging the Evolution of Nanoscale Photocurrent Collection and Transport Networks during Annealing of Polythiophene/Fullerene Solar Cells. Nano Lett. 2009, 9, 2946-2952.

(32) Sirringhaus, H.; Brown, P. J.; Friend, R. H.; Nielsen, M. M.; Bechgaard, K.; Langeveld-Voss, B. M. W.; Spiering, A. J. H.; Janssen, R. A. J.; Meijer, E. W. Microstructure--Mobility Correlation in Self-Organised, Conjugated Polymer Field-Effect Transistors. Synth. Met. 2000, 111-112, 129-132.

(33) Yang, C.; Orfino, F. P.; Holdcroft, S. A Phenomenological Model for Predicting Thermochromism of Regioregular and Nonregioregular Poly(3-Alkylthiophenes). Macromolecules 1996, 29, 6510-6517.

Theander, M.; Svensson, M.; Ruseckas, A.; Zigmantas, D.; Sundström, V.; Andersson, M. R.; Inganäs, O. High Luminescence from a Substituted Polythiophene in a Solvent with Low Solubility. Chem. Phys. Lett. 2001, 337, 277-283.

Clark, J.; Chang, J.-F.; Spano, F. C.; Friend, R. H.; Silva, C. Determining Exciton Bandwidth and Film Microstructure in Polythiophene Films Using Linear Absorption Spectroscopy. Appl. Phys. Lett. 2009, 94, 163306. 
(36) Boudouris, B. W.; Ho, V.; Jimison, L. H.; Toney, M. F.; Salleo, A.; Segalman, R. A. Real-Time Observation of Poly(3-Alkylthiophene) Crystallization and Correlation with Transient Optoelectronic Properties. Macromolecules 2011, 44, 6653-6658.

(37) Pingel, P.; Zen, A.; Abellón, R. D.; Grozema, F. C.; Siebbeles, L. D. A.; Neher, D. Temperature-Resolved Local and Macroscopic Charge Carrier Transport in Thin P3HT Layers. Adv. Funct. Mater. 2010, 20, 2286-2295.

(38) Gierschner, J.; Huang, Y.-S.; Van Averbeke, B.; Cornil, J.; Friend, R. H.; Beljonne, D. Excitonic versus Electronic Couplings in Molecular Assemblies: The Importance of Non-Nearest Neighbor Interactions. J. Chem. Phys. 2009, 130, 044105. 
For Table of Contents use only

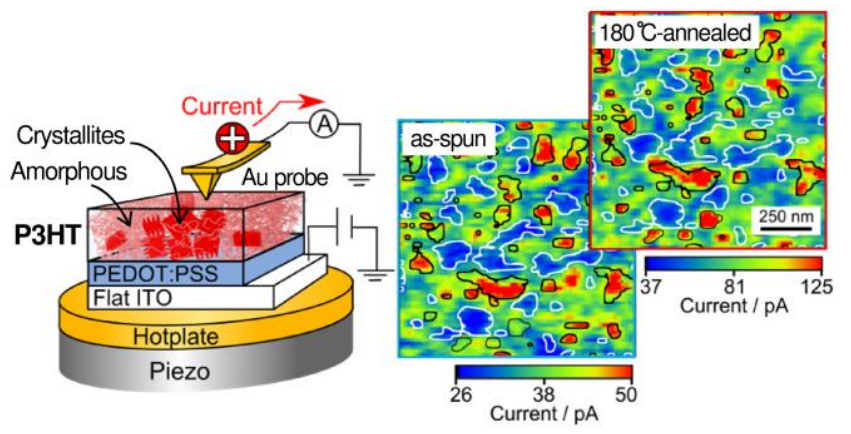

Nanostructures for Efficient Hole Transport in Poly(3-hexylthiophene) Film: A Study by Conductive Atomic Force Microscopy

Miki Osaka, Hiroaki Benten, Hideo Ohkita, Shinzaburo Ito, Hiroki Ogawa, and Toshiji Kanaya 